\title{
Hearing and Tinnitus Evaluation in Music Teachers
}

Selim Unsal ${ }^{1 *}$

Fatih $\mathrm{Bal}^{2}$

\begin{abstract}
Purpose: Music is an aesthetic whole consisting of sounds combined according to a certain purpose, method and understanding . Therefore, it also interacts with emotions in listeners. Music sounds are in the range of approximately $20-8000 \mathrm{~Hz}$. This frequency range is within the frequency spectrum of the human cochlea. The aim of this research is to evaluate the hearing thresholds of music teachers and to determine whether they cause tinnitus.

Material and Methods: This research was conducted in Turgut Özal University Medical Faculty Hospital Audiology and Speech Disorders clinic. A total of 35 people (17 music teachers, 18 control groups) participated in the research. In this research, 17 music teachers (11 females, 6 males) aged 24-39, and 18 volunteers (12 females, 6 males) participated in the control group. After the otoscopic examination of all participants, pure tone audiometry (in the range of $125-16,000 \mathrm{~Hz}$ ), immitansmetric examination and Distortion Product Otoacoustic Emission (DPOAE) tests were performed. Tinnitus Handicap Inventory (THI) was used in the evaluation of tinnitus.

Results: There was no statistically significant difference between the music teachers and the control group in all pure tone hearing threshold averages of $125-16,000 \mathrm{~Hz}(\mathrm{p}>0.05)$. The Uncomfortable Level (UCL) average of music teachers was $104.12 \pm 3.83 \mathrm{~dB}$ for the right ear, $108.33 \pm 3.83 \mathrm{~dB}$ for the control group, $107.78 \pm 4.28 \mathrm{~dB}$ for the left ear, and $103.53 \pm 4.28$ for the control group. DPOAE results were found statistically significant at $3000 \mathrm{~Hz}$ only for the right and left ears $(p=0.036 ; p=0.015$, respectively). Also, for DPOAE test, the control group's OAE values were higher than the music teachers. According to the Tinnitus Handicap Inventory in music teachers, 1 st degree tinnitus has emerged.
\end{abstract}

Conclusion: According to the findings obtained, the low UCL value in music teachers compared to the control group suggests the tolerance problem. Continuous exposure to the sound was thought to create a noise effect on the cochlea, and as a result, tinnitus susceptibility appeared in music teaching.

Keywords: Tinnitus; Music teachers, Hearing.

${ }^{1}$ Department of Department of Speech and Language Therapy, Faculty of Health Science İstanbul Atlas University, Turkey

${ }^{2}$ Department of Psychology, Faculty of Arts and Sciences, Sakarya University, Turkey

Department of Department of Speech and Language Therapy, Faculty of Health Science İstanbul Atlas University, Turkey. E-mail: selimunsal1@yahoo.com Phone: +90 5535938536

Paper submitted on March 08, 2021; and Accepted on April 30, 2021 


\section{INTRODUCTION}

Music can be defined as the art of combining sounds or musical notes with harmonious patterns that are pleasant and satisfy emotions. Music is also a melody ${ }^{1}$ In the last decade, there has been a marked increase in the number of people listening to music and the time spent listening to music. One of the most important reasons for this increase is the easy access to the instruments required for listening to music (advances in technology for personal music players and smartphones) $)^{2}$. In addition, listening to loud music can cause various hearing disorders, especially in adolescents and young adults ${ }^{3,4}$. Even listening to light music for 8-10 hours a day can cause hearing impairment.2 The World Health Organization (WHO) estimates that 1.1 billion young people and young adults are at risk of hearing loss due to exposure to entertainment noises such as music ${ }^{5}$. Listening to music is seen as a positive aspect of life in most cultures. However, exposure to loud noise causes hearing loss and is responsible for $16 \%$ of hearing loss worldwide 6 . Exposure to chronic noise, oxidative stress, metabolic exhaustion and ischemia also causes the internal and external hairy cells in the cochlea to gradually disappear 7,8 Those who are interested in professional music (music teachers) are at risk for hearing loss, tinnitus, hyperacusis and diplacusis. Tinnitus is defined as perception of sound without any external stimuli and affects $10-25 \%$ of the adult population ${ }^{9,10}$. Tinnitus negatively affects individuals' daily activities and quality of life. Hearing loss and exposure to loud sounds are among the risk factors of tinnitus. In individuals with hearing loss due to noise, tinnitus ranges between $35-77 \%{ }^{11,12}$. Hyperacusis is often a condition associated with tinnitus (Heller, 2003) ${ }^{13}$. It is defined as a reduced tolerance accompanied by painful sensitivity in perceptual, psychological and social dimensions, with sounds of average intensity, sometimes ordinary environmental sounds. Those dealing with professional music can be constantly exposed to loud sounds. This situation brings problems with the hearing system. The aim of this research is to evaluate the hearing thresholds of music teachers and to determine whether they cause tinnitus or hyperacusis.

\section{MATERIALS AND METHODS}

Participants: This study was conducted in the Audiology and Speech Disorders clinic of Turgut Ozal University Medical Faculty Hospital. Approval was obtained from the Non-Invasive Clinical Research Ethics Committee of Turgut Ozal University. A total of 35 people (17 music teachers, 18 control groups) participated in the study. In this study, 17 music teachers (11 females, 6 males) aged 24-39, and 18 volunteers (12 females, 6 males) participated in the control group. The ages of the individuals participating in this study range between 22 and 38 . The average age of music teachers is $26.40 \pm 5.6$, and the average age of control groups is $27.19 \pm 6.3$. Informed consent form was taken from all participants. After the otoscopic examination of all participants, pure tone audiometry (in the range of $125-16,000 \mathrm{~Hz}$ ), immitansmetric examination and Distortion Product Otoacoustic Emission (DPOAE) tests were performed. Tinnitus Handicap Inventory (THI) was used in the evaluation of tinnitus. Those with normal otoscopic examination for music teachers and control group were included in the study. Those who had any history of ear, acoustic trauma, ototoxic drug use, and neurological and psychiatric illness were excluded from the study.

Turkey's teachers are entering a total of 30 hours of classes per day 6 hours per week. Music teachers also attend classes in a similar way. The weekly hours of music teachers participating in the study vary between 25-30. Music teachers are also interested in music outside of school and this period varies between 8-10 hours weekly. In our study, these criteria were taken as basis to ensure standardization. In addition, the working time of the teachers who participated in the study as teachers varies between 10-13 years. There is also no acoustic feature (sound insulation in floors, ceilings and walls, etc.) especially in the classes where music teachers attend music lessons.

Audiological Evaluation: Hearing thresholds of all participants in the range of $0.125-16 \mathrm{kHz}$ were determined. Interacoustics AC-40 clinical audiometer (Assens, Denmark) was used to detect hearing thresholds and speech tests. Audiometric Earphones TDH-39P Telephonics are used for hearing thresholds between $0.125-8 \mathrm{kHz}$, and Koss HV-IA supraaural headphones are used for hearing thresholds between 10-16 $\mathrm{kHz}$. Speaking tests were done live by microphone. The Speech Reception Threshold (SRT), Words Discrimination Score (WDS), the Most Comfortable Level (MCL) and Uncomfortable Level (UCL) were determined. Whether or not middle ear functions are normal was determined by Interacoustic AZ-26 impedance meter (Assens, Denmark). Measurements were made between $+200 \mathrm{daPa}-400 \mathrm{daPa}$ pressure range, $226 \mathrm{~Hz}$ probe tone and $80 \mathrm{dBSPL}$. Distortion Product Otoacoustic Emission (DPOAE) measurements were made with Madsen Capella (GN Otometrics A/S 2630 Taastrup, Denmark). Measurements were made at the frequency of 2f1-f2 (f2/f1 ratio 1.22), at the level of $65 \mathrm{dBHL}$ (for L1) and $55 \mathrm{dBHL}$ (for L2). DPOAE measurements were made between $0.75 \mathrm{kHz}$ and 8 $\mathrm{kHz}$, and in industrial cabins, "Industrial Acoustics Company (IAC)". Signal Noise Ratio (SNR) ratio was recorded for all frequencies.

Tinnitus Handicap Inventory (THI): Tinnitus Handicap Inventory is a highly reliable and consistent survey used to measure the symptoms of patients with tinnitus. It was adapted to Turkish by Aksoy et al (2007) ${ }^{14}$. There are 25 questions in this questionnaire and the answers are three 
options: "Yes", "No" and "Sometimes". Yes answer is 4 , Sometimes answer is 2 , No answer is 0 points. The lowest 0 and the highest 100 points can be obtained. The evaluation according to the score result is shown in Table 1.

Statistical Analysis: SPSS program (IBM SPSS Statistics 21, SPSS inc., An IBM Co., Somers, NY) was used for statistical analysis. While evaluating the study data, descriptive statistical analyzes (mean, standard deviation) were performed and the appropriateness of the data to normal distribution was examined. Independent Samples $t$ test was used for those with normal distribution and Mann Whitney $U$ test was used for those with no normal distribution. When $p$ values are calculated less than 0.05 , it is considered statistically significant.

\section{RESULTS}

In this research, 17 music teachers (11 females, 6 males) aged 24-39, and 18 volunteers (12 females, 6 males) participated in the control group. The comparison of the hearing thresholds of the control group and music teachers participating in the research in $\mathrm{dB}$ (mean and standard deviation (SD)) is shown in Table 1. In the statistical evaluation, no difference was observed between the groups. It is noteworthy that although there is no statistical difference, the hearing thresholds of music teachers are higher than the control group. The results obtained from the speech tests (SRT, WDS, MCL and UCL) are shown in Table 2 . No statistically significant difference was found between the groups in SRT, MCL and WDS test results. However, UCL test results are statistically significant between groups. In the comparison of DPOAE test results (SNR), it was observed that only $3000 \mathrm{~Hz}$ was statistically significant. At other frequencies, the overall height of the SNR values of the control group is remarkable. At the same time, DPOAE reproducibility values of the control group were higher than the music teachers Table 3 .

The evaluation results with Tinnitus Handicap Inventory are shown in Table 4. None of the participants in the control group reported tinnitus complaints. The participants in the music teachers group received an average of $12.42 \pm 3.26$ points from the test consisting of 25 questions and a total score of 100 .

Table 1: Comparison of Music Teachers and Control Group's Hearing Thresholds.

\begin{tabular}{cccc}
\hline Frequency & $\begin{array}{c}\text { Control Group } \\
\text { (Mean } \pm \text { SD) }\end{array}$ & $\begin{array}{c}\text { Music Teachers } \\
\text { (Mean } \pm \text { SD) }\end{array}$ & p evaluate \\
\hline Right $125 \mathrm{~Hz}$ & $8.33 \pm 4.2$ & $6.47 \pm 4.2$ & 0.106 \\
Right $250 \mathrm{~Hz}$ & $6.94 \pm 4.89$ & $4.41 \pm 3.89$ & 0.075 \\
Right $500 \mathrm{~Hz}$ & $7.5 \pm 5.22$ & $8.24 \pm 5.22$ & 0.889 \\
Right $1000 \mathrm{~Hz}$ & $7.5 \pm 3.09$ & $5.59 \pm 3.09$ & 0.055 \\
Right $2000 \mathrm{~Hz}$ & $8.33 \pm 4.85$ & $5.88 \pm 4.85$ & 0.069 \\
Right $4000 \mathrm{~Hz}$ & $9.72 \pm 5.28$ & $7.35 \pm 5.28$ & 0.196 \\
Right $6000 \mathrm{~Hz}$ & $9.44 \pm 6.39$ & $7.06 \pm 6.39$ & 0.250 \\
Right $8000 \mathrm{~Hz}$ & $15.56 \pm 9.22$ & $13.22 \pm 8.53$ & 0.617 \\
Right $12.000 \mathrm{~Hz}$ & $15.28 \pm 13.11$ & $13.82 \pm 13.11$ & 0.482 \\
Right $16.000 \mathrm{~Hz}$ & $21.67 \pm 18.55$ & $25 \pm 18.55$ & 0.595 \\
Left $125 \mathrm{~Hz}$ & $6.94 \pm 3.04$ & $6.76 \pm 3.04$ & 0.746 \\
Left $250 \mathrm{~Hz}$ & $6.39 \pm 4.13$ & $5.88 \pm 4.13$ & 0.717 \\
Left $500 \mathrm{~Hz}$ & $5.56 \pm 4.5$ & $7.65 \pm 4.5$ & 0.081 \\
Left $1000 \mathrm{~Hz}$ & $7.5 \pm 4.29$ & $5.88 \pm 4.29$ & 0.160 \\
Left $2000 \mathrm{~Hz}$ & $6.11 \pm 4.39$ & $4.12 \pm 4.39$ & 0.149 \\
Left $4000 \mathrm{~Hz}$ & $8.33 \pm 5.14$ & $8.24 \pm 5.14$ & 0.961 \\
Left $6000 \mathrm{~Hz}$ & $7.5 \pm 6.24$ & $9.12 \pm 6.24$ & 0.550 \\
Left $8000 \mathrm{~Hz}$ & $12.22 \pm 8.61$ & $10.88 \pm 8.61$ & 0.679 \\
Left $12.000 \mathrm{~Hz}$ & $12.5 \pm 10.18$ & $16.47 \pm 10.18$ & 0.497 \\
Left $16.000 \mathrm{~Hz}$ & $19.72 \pm 15.29$ & $23.24 \pm 15.29$ & 0.525 \\
& & &
\end{tabular}

Table 2: The results of speaking tests.

\begin{tabular}{cccc}
\hline Tests & $\begin{array}{c}\text { Control Group } \\
\text { (Mean } \pm \text { SD) }\end{array}$ & $\begin{array}{c}\text { Music Teachers } \\
\text { (Mean } \pm \text { SD) }\end{array}$ & p evaluate \\
Right SRT & $9.44 \pm 2.91$ & $8.53 \pm 2.91$ & 0.465 \\
Left SRT & $9.44 \pm 2.91$ & $8.53 \pm 2.91$ & 0.465 \\
Right WDS & $100 \pm 0.0$ & $100 \pm 0.0$ & statistical evaluation was not done. \\
Left WDS & $100 \pm 0.0$ & $100 \pm 0.0$ & statistical evaluation was not done. \\
Right MCL & $49.44 \pm 2.91$ & $48.53 \pm 2.91$ & 0.465 \\
Left MCL & $49.44 \pm 2.91$ & $48.53 \pm 2.91$ & 0.465 \\
Right UCL & $108.33 \pm 2.91$ & $104.12 \pm 3.83$ & $0.009^{\star}$ \\
Left UCL & $107.78 \pm 4.28$ & $103.53 \pm 4.28$ & $0.010^{*}$ \\
\hline
\end{tabular}


Table 3: Comparison of DPOAE results.

\begin{tabular}{cccc}
\hline Frequency & $\begin{array}{c}\text { Control Group } \\
\text { (Mean } \pm \text { SD) }\end{array}$ & $\begin{array}{c}\text { Music Teachers } \\
\text { (Mean } \pm \text { SD) }\end{array}$ & p evaluate \\
Right $750 \mathrm{~Hz}$ & $4.2 \pm 3.5$ & $4 \pm 3.5$ & 0.854 \\
Right $1000 \mathrm{~Hz}$ & $7.4 \pm 3$ & $7.5 \pm 3$ & 0.934 \\
Right $1500 \mathrm{~Hz}$ & $11.9 \pm 4.2$ & $8.9 \pm 2.2$ & 0.827 \\
Right $2000 \mathrm{~Hz}$ & $10.5 \pm 3.2$ & $9.7 \pm 3.2$ & 0.608 \\
Right $3000 \mathrm{~Hz}$ & $12.7 \pm 3.9$ & $9.6 \pm 3.9$ & $\mathbf{0 . 0 3 6 ^ { * }}$ \\
Right $4000 \mathrm{~Hz}$ & $14.9 \pm 4.5$ & $16.5 \pm 4.5$ & 0.180 \\
Right $6000 \mathrm{~Hz}$ & $11.5 \pm 6.9$ & $13 \pm 6.9$ & 0.504 \\
Right $8000 \mathrm{~Hz}$ & $12.6 \pm 6.6$ & $11.6 \pm 6.6$ & 0.624 \\
Left $750 \mathrm{~Hz}$ & $5 \pm 3.8$ & $5 \pm 3.8$ & 0.990 \\
Left $1000 \mathrm{~Hz}$ & $6.7 \pm 3.1$ & $7.1 \pm 3.1$ & 0.784 \\
Left $1500 \mathrm{~Hz}$ & $7.5 \pm 2.8$ & $7.8 \pm 2.8$ & 0.823 \\
Left $2000 \mathrm{~Hz}$ & $13 \pm 3.7$ & $6.9 \pm 3.7$ & 0.180 \\
Left $3000 \mathrm{~Hz}$ & $11.9 \pm 4.1$ & $8 \pm 4.1$ & $\mathbf{0 . 0 1 5 ^ { \star }}$ \\
Left $4000 \mathrm{~Hz}$ & $13.4 \pm 5.4$ & $11.7 \pm 5.4$ & 0.380 \\
Left $6000 \mathrm{~Hz}$ & $16.9 \pm 5.2$ & $11.8 \pm 5.2$ & 0.716 \\
Left $8000 \mathrm{~Hz}$ & $12.4 \pm 4.7$ & $10.1 \pm 4.7$ & 0.181 \\
\hline
\end{tabular}

Table 4: Tinnitus Handicap Inventory (TDI) Evaluation.

\begin{tabular}{ccc} 
Groups & Average score & Evaluation \\
Control Group & 0 & No one with tinnitus \\
Music Teachers & $12.42 \pm 3.26$ & Slight (only heard in quiet environments) \\
\hline
\end{tabular}

\section{DISCUSSION}

When music is the dominant sound source, the resulting auditory conditions are called Music Induced Hearing Disorders (MIHD). The marked increase in prevalence of MIHDs in music industry professionals as compared to the general population indicates that music should be considered a potentially damaging stimulus. Music differs from noise in both acoustical and psychophysical qualities. Even within music professionals' subgroups, variability in occupational settings, work schedules, and dominate sound sources further complicates risk criteria generalizations of music industry professionals Koelsch et. al. (2005) ${ }^{15}$ investigated the functional neuroanatomy of music perception in children and adults. As a result of the research, they stated that music studies in children and adults caused strong activations in the frontal and temporal anterior regions of the brain ${ }^{16}$ According to the studies, children educated at an early age with music become more comfortable, peaceful, mild and selfconfident. It is known that music has a positive effect on fields such as language, mathematics, working memory, focus and attention and increases success ${ }^{17}$. There are cases where music is harmful besides this benefits (such as hearing loss). Just like noise-related hearing losses, music-induced hearing losses occur in the region of $3000-6000 \mathrm{~Hz}$ notch ${ }^{18}$. Affection in music-related hearing loss is bilateral and symmetrical ${ }^{19}$ Music professionals may not be able to continue their careers due to hearing problems arising from music. In addition, hearing loss, tinnitus, hyperacusis, diplacusis and tolerance problems may occur in these people ${ }^{6-15}$. In this research, we compared the music thresholds and hearing thresholds of people who did not deal with music (control group),
SRT, WDS, MCL, UCL and DPOAE test results. We could not find a statistically significant difference between the hearing thresholds, SRT, MCL WDS test results of the music teachers and the control group. However, it was thought that the hearing thresholds of music teachers increased compared to the control group, and this occurred as a result of continuous exposure to music. A statistically significant difference was found between the UCL test results between the groups ${ }^{20}$. This is called the tolerance problem. Music teachers are uncomfortable with loud sound. These results show that cochlear sensitivity develops in music teachers. In other words, it suggests that there is an effect on the outer and inner hair cells. In DPOAE test, a statistically significant difference was found between the groups at bilateral $3000 \mathrm{~Hz}$. It is noteworthy that there is a significant difference in $3000 \mathrm{~Hz}$. Depending on the noise, temporary or permanent threshold changes are observed in the cochlea.20 Affecting the cochlea at the low level of noise occurs at the stimulus frequency, and at high intensity levels at the 1 octave frequency of the frequency that stimulates threshold changes ${ }^{21-22}$. Sataloff stated that, depending on the frequency spectrum, the first signs of noise-induced hearing loss started on the audiogram as "V" at a maximum of $2-6 \mathrm{kHz}{ }^{23}$. This explains the difference in emission values of $3000 \mathrm{~Hz}$ in DPOAE test results in music teachers. In other words, the high intensity music noise at the frequency of $2000 \mathrm{~Hz}$ may have affected the $3000 \mathrm{~Hz}$ region. As can be seen, although music is a pleasant melody, it can negatively affect people's hearing health. Plontke et. al. (2004) ${ }^{24}$ stated that the noise in musical instruments is at the level of 75-105 dB (A). They reported that sounds more severe than $75 \mathrm{~dB}(\mathrm{~A})$ negatively affect the human body, especially the hearing system ${ }^{24}$. Fligor and Cox (2004) 
stated that exposure to loud noise or continuous music is a risk factor for noise-induced hearing loss Couth et. al. (2019) ${ }^{25}$ suggested the protection of hearing health in music environments ${ }^{26}$.

In this research, tinnitus evaluation was done with Tinnitus Handicap Inventory (THI). In the evaluation of tinnitus, it was observed that music teachers experienced "slight level" tinnitus. Tinnitus is thought to be due to the effect of the inner ear (tolerance problem). Music teachers who participated in this research have been working for approximately 10 years. It is estimated that they will experience more hearing impairment and suffer more from tinnitus in the years to come. Chronic disturbing tinnitus can significantly reduce the confidence in musicians' auditory perceptual abilities in listening situations and prevent both focus and personal enjoyment for performance-based roles. 27 In addition, it can cause conditions such as hyperacusis (discomfort from non-loud sound), diplacusis (inter-ear frequency difference) and dysacusis (perception of distorted sound in only one ear). Beyond the effects, the development of music-related hearing loss also creates a psychological barrier for a music industry professional; the person who no longer feels comfortable relying on his "ear" may not be able to pursue a career in music. Even if there is no significant hearing loss in individuals who have been teaching music for about 10 years, there is a significant difference in cochlear sensitivity and $3 \mathrm{kHz}$, one of the frequencies affected by noise, compared to the control group with normal hearing. It was observed. This made us think that music has a noise effect in the cochlea. We think that the findings found in our study may be helpful in early diagnosis of music-related hearing loss in this group.

\section{CONCLUSION}

Music professionals are constantly exposed to music. Accordingly, sensitivity develops in the inner ears. Depending on this sensitivity, tinnitus may occur. Tinnitus negatively affects auditory perception and people who are psychologically interested in music. Music professionals are not a homogeneous group spread across the population, it is necessary to educate this group on music-related hearing disorders and to carry out public health campaigns.

\section{CONFLICT OF INTEREST}

The Author declares no potential conflict of interest on publishing this paper.

\section{REFERENCES}

1. Sheriff A. Music and its Effects. Scotts Valley, CA: Create Space Independent Publishing Platform. 2014.

2. Manchaiah V, Zhao F, Ratinaud P. Young Adults' Knowledge and Attitudes Regarding "Music" and "Loud Music" Across Countries: Applications of Social Representations Theory. Front Psychol. 2019;25:139-40.

3. Zhao F, Manchaiah VK, French D, Price SM. Music exposure and hearing disorders: an overview. Int J Audiol. 2010;49:54-64.
4. Jiang W, Zhao F, Guderley N, Manchaiah V. Daily music exposure dose and hearing problems using personal listening devices in adolescents and young adults: $A$ systematic review. Int J Audiol. 2016;55:197-205.

5. World Health Organization. Billion People are at Risk of Hearing Loss. 2015;1:6-100.

6. Di Stadio A, Dipietro L, Ricci G, Della Volpe A, Minni A, Greco A, et al. Hearing Loss, Tinnitus, Hyperacusis, and Diplacusis in Professional Musicians: A Systematic Review. Int J Environ Res Public Health. 2018;15:120-22.

7. Fetoni AR, Garzaro M, Ralli M, Landolfo V, Sensini M, Pecorari $G$, et al. The monitoring role of otoacoustic emissions and oxidative stress markers in the protective effects of antioxidant administration in noise-exposed subjects: a pilot study. Med Sci Monit. 2009;15:1-8.

8. Henderson D, Bielefeld EC, Harris $\mathrm{KC}, \mathrm{Hu} \mathrm{BH}$. The role of oxidative stress in noise-induced hearing loss. Ear Hear. 2006;27:1-19.

9. Baguley D, McFerran D, Hall D. Tinnitus. Lancet. 2013;382:1600-7.

10. Langguth B, Kreuzer PM, Kleinjung T, De Ridder D. Tinnitus: Causes and clinical management. Lancet Neurol. 2013;12:920-30.

11. Mrena R, Savolainen S, Kuokkanen JT, Ylikoski J. Characteristics of tinnitus induced by acute acoustic trauma: A long-term follow-up. Audiol. Neurootol. 2002;7:122-130.

12. Nageris BI, Attias J, Raveh E. Test-retest tinnitus characteristics in patients with noise-induced hearing loss. Am. J. Otolaryngol. 2010;31:181-4.

13. Aazh H, McFerran D, Salvi R, Prasher D, Jastreboff $M$, Jastreboff $P$. Insights from the First International Conference on Hyperacusis: causes, evaluation, diagnosis and treatment. Noise Health. 2014;16:123-6.

14. Aksoy S, Firat $Y$, Alpar R. The Tinnitus Handicap Inventory: a study of validity and reliability. Int Tinnitus J. 2007;13:94-8.

15. Wartinger F, Malyuk H, Portnuff CDF. Human exposures and their associated hearing loss profiles: Music industry professionals. J Acoust Soc Am. 2019;146:390-6.

16. Koelsch S, Fritz T, Schulze K, Alsop D, Schlaug G. Adults and children processing music: an fMRI study. Neuroimage. 2005; 25:1068-76.

17. Atherton RP, Chrobak QM, Rauscher FH, Karst AT, Hanson MD, Steinert SW, Bowe KL. Shared Processing of Language and Music. Exp Psychol. 2018;65:40-8.

18. Royster JD, Royster LH, Killion MC. Sound exposures and hearing thresholds of symphony orchestra musicians. J Acoust Soc Am. 1991;89:2793-803.

19. Chasin M. Musicians and the Prevention of Hearing Loss (Singular Publishing, San Diego, CA). 1996;2:1-6.

20. Mills JH, Gilbert RM, Adkins WY. Temporary threshold shifts in humans exposed to octave bands of noise for 16 to 24 hours. J Acoust Soc Am. 1979;65:1238-48.

21. Schmiedt RA. Acoustic injury and the physiology of hearing JAcoust Soc Am. 1984;76:1293-1317.

22. Ward WD. Adaptation and Fatique. In Jerger, J. edition. Modern Developments in Audiology. 2nd. Edition. New York: Academic Press. 1973;1:241-86. 
23. Sataloff RT, Sataloff J. Occupational Hearing Loss. $3^{\text {rd }}$ edition. (Taylor and Francis Publishin, Newyork). 2006;1:1-26.

24. Plontke S, Zenner HP. Current aspects of hearing loss from occupational and leisure noise. GMS Curr Top Otorhinolaryngol Head Neck Surg. 2004;3:200-4.

25. Fligor BJ, Cox LC. Output levels of commercially available portable compact disc players and the potential risk to hearing. Ear Hear. 2004;25:513-27.
26. Couth S, Mazlan N, Moore DR, Munro KJ, Dawes P. Hearing Difficulties and Tinnitus in Construction, Agricultural, Music, and Finance Industries: Contributions of Demographic, Health, and Lifestyle Factors. Trends Hear. 2019;23:23-31.

27. Vogel I, van de Looij-Jansen PM, Mieloo CL, Burdorf A, de Waart F. Risky music listening, permanent tinnitus and depression, anxiety, thoughts about suicide and adverse general health. PLoS One. 2014;9:e989-12. 